\title{
Study on rock breaking mechanism of PDC bit with rotating module
}

\author{
Kuilin Huang ${ }^{\mathrm{a}}$,Yingxin Yang ${ }^{\mathrm{a}, \mathrm{b}, \mathrm{c} *}$,Yang Liu ${ }^{\mathrm{d}}$,Yunpeng Zhong ${ }^{\mathrm{a}}$, Haitao Ren $^{\mathrm{a}}$ \\ a Collge of Mechanical and Electrical Engineering,Southwest Petroleum University,Chengdu P.R.China,610500 \\ b.State Key Laboratory of Oil and Gas Reservoir Geology and Development Engineering, Chengdu P.R.China,610500 \\ c.Drilling Research Institute of National Engineering Laboratory for Oil and Gas Drilling Technology, Chengdu P.R.China ,610500 \\ d College of Engineering、Mathematics and Physical Sciences University of Exeter, Exeter, UK,EX4 4QF
}

\section{Highlights:}

1. A rotating modular PDC bit is proposed, the new bit realizes local cross scraping and improves rock breaking efficiency;

2 Through unit experiments, the author studies the changing rules of the rotation speed and force of the rotating module with the module's the journal angle and the side angle.

3. Compared with the conventional PDC bit, the rate of penetration (ROP)of rotating modular PDC bit is increased by $15 \%$ and the Mechanical specific energy(MSE)is reduced by $14 \%$.

Abstract: In view of the problems of low ROP, short bit life and high energy consumption of bits in deep and difficult formations. Basing on the $360^{\circ}$ rotating teeth and disc PDC bit, a rotating modular PDC bit is proposed. The rock breaking efficiency of the bit is improved by "cross scraping" of the rotating module element (RME) and fixed cutting teeth. In addition, the rotating module element works alternately, which cools the cutting teeth in time, slowing the wear of the cutting teeth and prolongs bit life. This paper introduces the structural characteristics and working principle of the novel bit, and carries out variable parameter experiments on the rotating module element (side angle, journal angle, and tooth arrangement density, etc.) The experimental results show that the rotating speed of the rotating module element increases with the increase of the side angle, and decreases with the increase of the axial angle. The variation rules of cutting load and MSE of rotating module element under different structural parameters are studied. The indoor test shows that the ROP of rotary modular PDC bit is increased by 15\% and the MSE is reduced by 14 $\%$, It is implies that the "cross scraping" of the rotary module element and the fixed cutting teeth can reduce the rock breaking specific work, and provide theoretical basis and support for the design of the subsequent rotary modular PDC bit.

Key word: Rotating module; Rock breaking mechanism; Alternate work

\section{Introduction}

For deep well and ultra deep well, the drilling cost of deep formation accounts for a considerable proportion in the total drilling cost(Zhu,H.Y.et al., 2019; Dan Scott. 2015). The low drilling rate of deep formation has seriously affected the well construction period and drilling cost of deep well and ultra deep well. Polycrystalline diamond compact bit (PDC bit) is the most important type of bit in oil and gas drilling. Its drilling footage has reached more than $92 \%$ of the global total drilling footage(Luc Perneder et al.,2012; H. Santos et al.,2000). For PDC bit, cutting tooth technology and bit structure form are important factors affecting rock breaking efficiency of PDC bit(Jiang, M.H et al.,2009; Zhu H.Y.et al., 2015). In order to improve the rock breaking efficiency and service life of the bit, scholars at home and abroad have carried out a lot of relevant research, among which the disc PDC bit with cross scraping is a composite bit which combines the fixed cutting structure with the cutting structure of the disc cutter, The offset angle of the disc cutter on the cross-cut PDC bit is very large. When the drill bit is rotated and drilled, the disc cutter rotates 
at a low speed. After the cutting teeth on the disc cutter are slowly pressed into the rock, a longer circumferential slip and radial slip are performed on the rock, and then the rock at the bottom of the hole is slowly cut out to form a spiral scraping trajectory. The teeth alternately scrape the broken rock at the bottom of the well in a slow alternating pattern. This spiral scraping trajectory overlaps with the concentric circle trajectory of the fixed cutting teeth of the fixed cutting structure. (Yang,Y.X. et al., 2017; Yang,Y.X. et al., 2018). In the drilling process, the cutting teeth on the disc cutter work slowly and slowly alternately to reduce the thermal wear. At the same time, the way of cross scraping is conducive to cutting teeth into the formation But the cutterhead cutting structure needs an independent support, which occupies the precious space of tooth distribution on the bit. For $360^{\circ}$ rotary bit, the cutting teeth can rotate freely in the process of rock breaking, which can avoid the occurrence of cutting eccentric wear and prolong the cutting life of the cutting teeth(Li,M., 2017; Li,M. et al., 2017). The application of rotary tooth technology in the development of Saudi Arabia's natural gas has significantly increased the bit penetration and drilling speed, When the rotary bit drilled into sandstone, it achieved a record of $160.2 \mathrm{~m}$, and the ROP reached $4.53 \mathrm{~m} / \mathrm{h}$. The ROP was increased by $76 \%$ compared to the maximum ROP of ordinary PDC bits in the same well section(Platt J, et al., 2016).However, due to the limitation of the size of the rotating teeth, the rotating axis of the teeth is too small, so the ability of the cutting teeth to bear the impact load is weak(Mostofi M. et al., 2013).

On the basis of $360^{\circ}$ rotating teeth and disc PDC bits, a rotating modular PDC bit is proposed in this paper. As shown in Fig. 1, the module teeth are used to work in turn slowly, so that the cutting teeth can be sufficiently cooled, and the cutting teeth on the module can be evenly worn, thus prolonging the service life of the cutting teeth. At the same time, in the radial coverage area of the module, the rotating module teeth and the fixed cutting teeth intersect By scraping and cutting the broken rock with fork, the protruding rock ridge can be formed naturally, which can improve the invasion ability of cutting teeth and the rock breaking efficiency of bit.

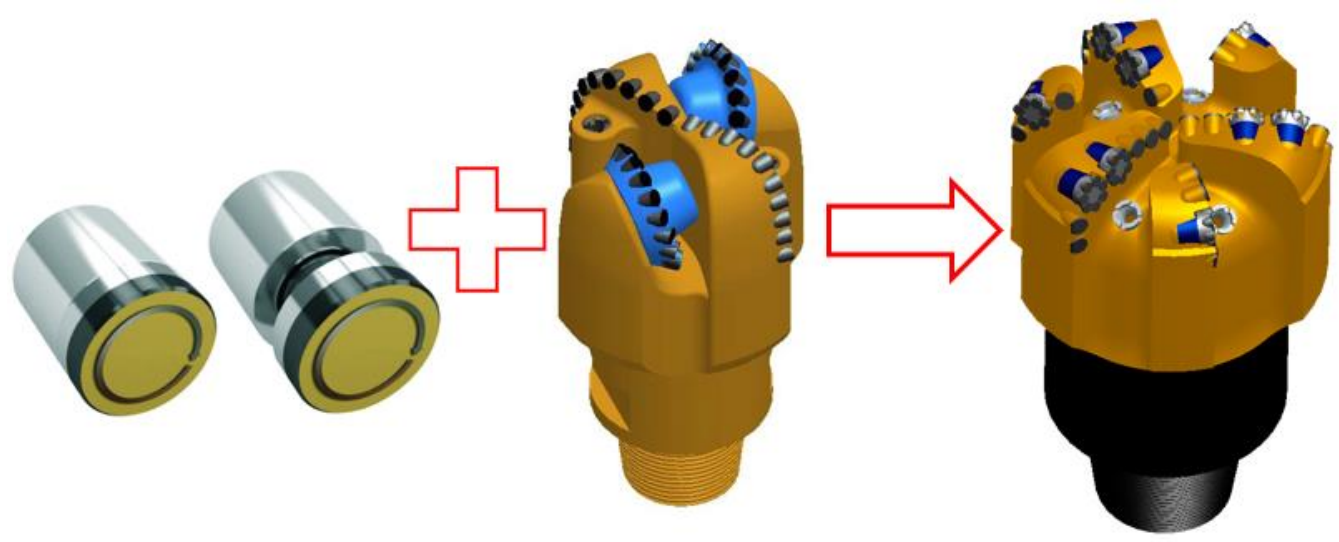

Fig. 1 Rotary modular PDC bit

\section{Structure characteristics and working mechanism of PDC bit with}

\section{rotating module}

\subsection{Structural features of rotary modular PDC bit}

The difference between the rotary modular PDC bit (as shown in Fig. 2) and the conventional PDC bit is that the rotary modular cutting element is introduced into the cutting wing of the fixed 
tooth PDC bit. The rotary modular cutting element is composed of the matrix (the matrix and the shaft are integrated), the cutting teeth, the shaft sleeve, etc., which organically combines the dynamic and static cutting structures to realize the local cross rock breaking and give full play to the advantages of PDC tooth rock breaking Each cutting tooth on the matrix alternately scrapes the rock to achieve uniform wear in each area of the same cutting module element; each cutting tooth interacts with the rock briefly and discontinuously during the working process, with small heat output and timely heat dissipation, which is not easy to generate thermal wear, so as to extend the life of the drill head. For the cutting teeth with small diameter, the contact area between a single tooth and the rock is small and the rock breaking ratio is small High pressure, easy to eat into the rock, the rotating pair of the rotating module element is set between the base body and the shaft sleeve, and there is no need to specially set the bearing support or bearing structure on the blade body, so that the structure of the drill bit is more simple, compact and reliable.

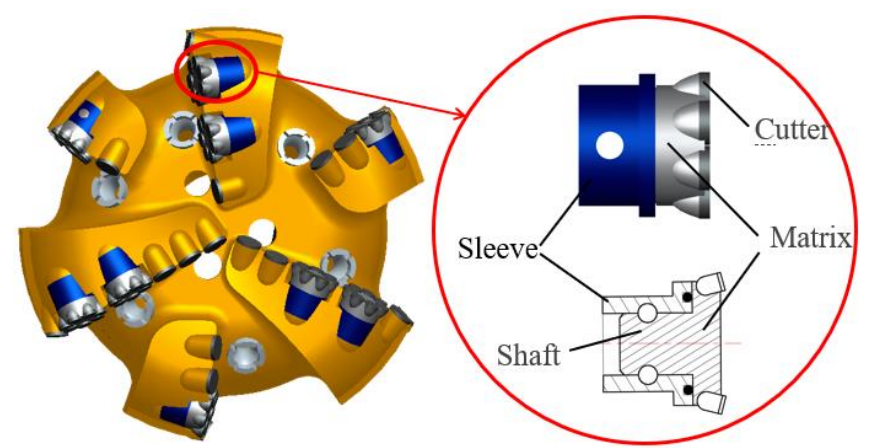

Fig. 2 Structure diagram of rotary modular PDC bit

\subsection{Working mechanism of rotary modular PDC bit}

When the rotary module element is set with a certain side angle, the teeth on the module element interact with the rock during the drilling process. Due to the existence of the side angle, the moment generated when the module rotates is large enough to overcome the resistance of the rock, then the module element will rotate, and the corresponding structural parameters of the module element (journal angle $\alpha$, side angle $\beta$, positioning radius $S$ ) As shown in Fig. 3. The module element rotates under the drive of rock, and the trace scraped at the bottom of the well is helix, because the module has circumferential slip and radial slip, which makes the cutting teeth in this area (assuming that the module is arranged in the outer $1 / 3$ area of the bit crown) and the fixed cutting teeth form a local cross scraping (schematic diagram of bottom hole track, as shown in Fig. 3 (c)), where the red track is the rotating die This kind of rock breaking method can significantly improve the rock breaking efficiency of the bit.

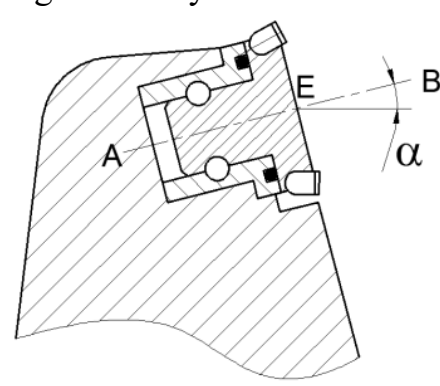

(a)The journal angle of module element

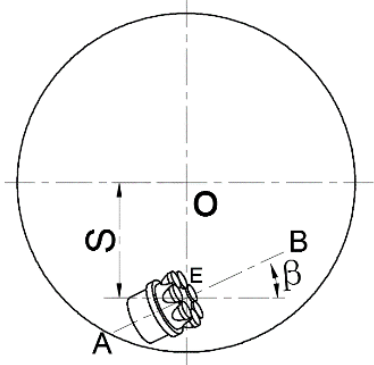

(b) The side angle and positioning radius of module element

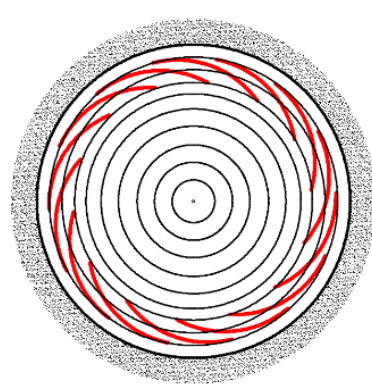

(c)The schematic diagram of well bottom track

Fig. 3 Structural parameters and bottom hole diagram of module element 
Whether the rotating module can rotate depends on whether the driving torque can overcome the friction of the bearing and the force of some module teeth breaking the rock. The driving force of module element rotation comes from the component of tangential force generated by the interaction between module element and rock in the direction of side angle. As shown in Fig. 4, the component force $f_{t} \sin \beta$ of the tangential force $F_{t}$ along the side angle direction is the driving force $F_{0}$ of the module; because of the existence of the axial inclination, the axial force $F_{N}$ and the tangential force $F_{t}$ generate the component forces $F_{n} \sin \alpha$ and $F_{t} \cos \beta \sin \alpha$ along the module axial direction, and the component forces $F_{n} \cos \alpha$ and $F_{t} \cos \beta \sin \alpha$ along the module element radial direction opposite.

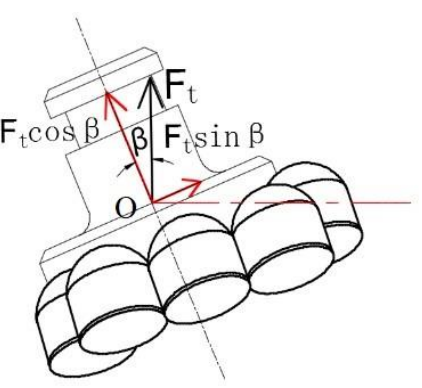

(a)Schematic diagram of tangential force received by the module

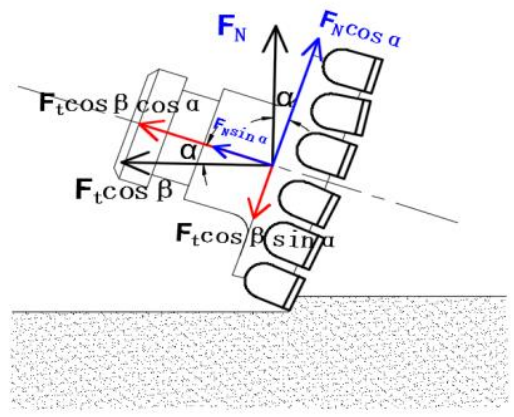

(b) Schematic diagram of component force

produced by the module due to axle inclination

Fig. 4 Force diagram of rotating module element

Because the bottom contact of module element interacting with rock is a dynamic process, this paper discusses the critical state of module element rotation, taking the alternative cutting of one tooth and two teeth as the research object. In an ideal state, if the bearing friction is small enough, the friction force to be overcome when the cutting teeth intrude into the rock will be ignored. At this time, the critical tooth arrangement of the teeth is shown in Fig. 5 (b); the corresponding structural parameters of the module element are shown in Fig. 5 (a), the diameter of the module element $D_{l}$, the diameter of the module element working contour (outer circle diameter) $D_{2}$, the diameter of the module element small teeth $D$, and the module element teeth The center distance of is $L$ (that is, the distance between the centers of teeth of two adjacent modules). According to fig. 5 (a), the center distance $L_{0}$ between $\mathrm{A}$ and $\mathrm{C}$ in Figure 5 (b) is:

$$
L_{0}=2 \sqrt{D_{1}^{2}-\left(D_{1}-h\right)^{2}}
$$

Where, $h$ cutting depth, $D_{l}$ rotating module diameter.

Therefore, in an ideal state, the module can keep continuous rotation as long as the tooth spacing $L$ of the module cutting teeth is less than $L_{0}$ and the side angle $\beta>0$. However, when the actual module works, it needs to overcome the friction of the bearing and the force of some broken rocks. As shown in Fig. 5, this paper takes one and two module teeth working alternately at the bottom of the well as the research object. 


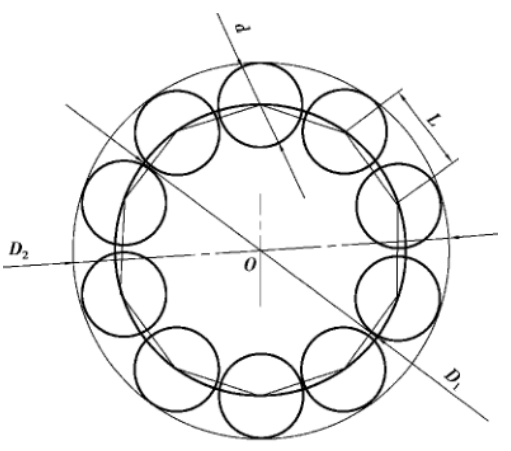

(a)Module size and structure parameter

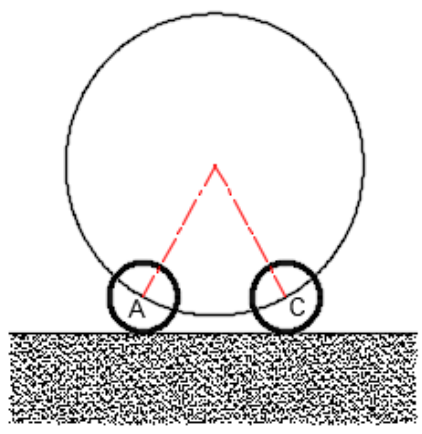

(b) Critical gear arrangement under ideal condition

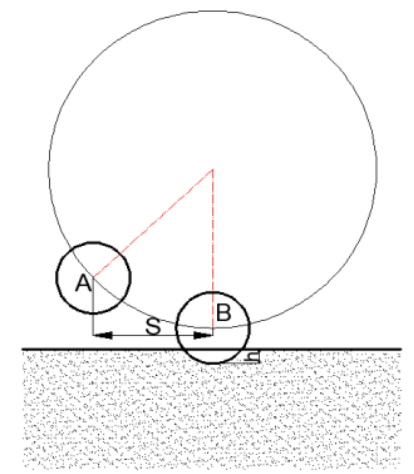

(c) Actual working state of module element

Fig. 5 Schematic diagram of module element cutting

When the module element rotates from A to B, the displacement of the module in the circumferential direction is $S$, and the displacement in the cutting depth direction is $h$. at this time, the module overcomes the force of the broken rock, and $f_{0}$ is an instantaneous force. With the increase of the cutting depth and the circumferential displacement, $f_{0}$ gradually increases; with the increase of the cutting depth, the contact area $S_{0}$ between the module teeth and the rock gradually increases, so does the tangential force and the driving force In this paper, the tangential force and the contact area between the module element and the rock are considered to be linear:

$$
F_{t}=k \cdot S_{0}
$$

Where, $k$ is the contact area coefficient of rock and module element; $S_{0}$ is the contact area.

As shown in Figure 5 (b), if the rotating module element is to work continuously, it is necessary to make the driving force $F_{t}$ generated when the cutting tooth $\mathrm{C}$ interacts with the rock greater than the resistance $f_{0}$ of the cutting tooth A invading the rock and the friction $f_{l}$ generated by the bearing. For the friction force $f_{1}$ generated by the bearing is related to the friction coefficient $\mu$ and positive pressure $N$ of the bearing, the friction force $f_{1}$ generated by the bearing is:

$$
f_{1}=u \cdot\left(N_{1}+N_{2}\right)
$$

Where $\mu$ is the friction coefficient between bearings, $N_{l}$ is the positive pressure at the shaft diameter, and $N_{2}$ is the positive pressure inside the bearing.

$$
\left\{\begin{array}{l}
N_{1}=F_{t} \cdot \cos \beta \cdot \cos \alpha+F_{N} \cdot \sin \alpha \\
N_{2}=F_{N} \cdot \cos \alpha-F_{t} \cdot \cos \beta \cdot \sin \alpha
\end{array}\right.
$$

$$
f_{1}=u \cdot\left(F_{t} \cdot \cos \beta \cdot \cos \alpha+F_{N} \cdot \sin \alpha+F_{N} \cdot \cos \alpha-F_{t} \cdot \cos \beta \cdot \sin \alpha\right)
$$

Therefore, as long as $F_{0}>f_{0}+f_{1}$ module can maintain continuous rotation state. Generally, the resistance $f_{0}$ of the cutting teeth when they just intrude into the rock is very small, which is ignored in the ideal state. The critical state in Fig. $5(\mathrm{~b})$ is $F_{0}>f_{1}$.

\section{Cutting experiment of rotating module element}




\subsection{Purpose of the experiment}

The working state of the rotating module element is affected by many factors, including the side angle, the journal angle, the number of teeth of the rotating module element and so on. The main purpose of the experiment is to find out the influence rules of various factors on the working state of the rotating module. Through the experiment of the rotating module element, the working state and rock breaking law of the rotating module element are studied, which provides the theoretical basis for the subsequent design of the new PDC bit.

\subsection{Experimental equipment and rock samples}

The rotating module element scraping experimental equipment includes a shaper testing machine, a three-way force sensor, a rotating module and a data acquisition system (as shown in Fig. 6).

In the experiment, the cutting element is the rotating module element, and the cemented carbide tooth (its model is YG15) is used. The cemented carbide has high hardness, strength and wear resistance. The diameter $D$ of the rotating module tooth is $8 \mathrm{~mm}$. The cemented carbide cutting elements, matrix and shaft sleeve together constitute the rotating module cutting element, as shown in Fig. 7. In the scraping test, the size of sandstone is $300 \mathrm{~mm} \times 250 \mathrm{~mm} \times 250 \mathrm{~mm}$, and the rock mechanical parameters are shown in Table 1.

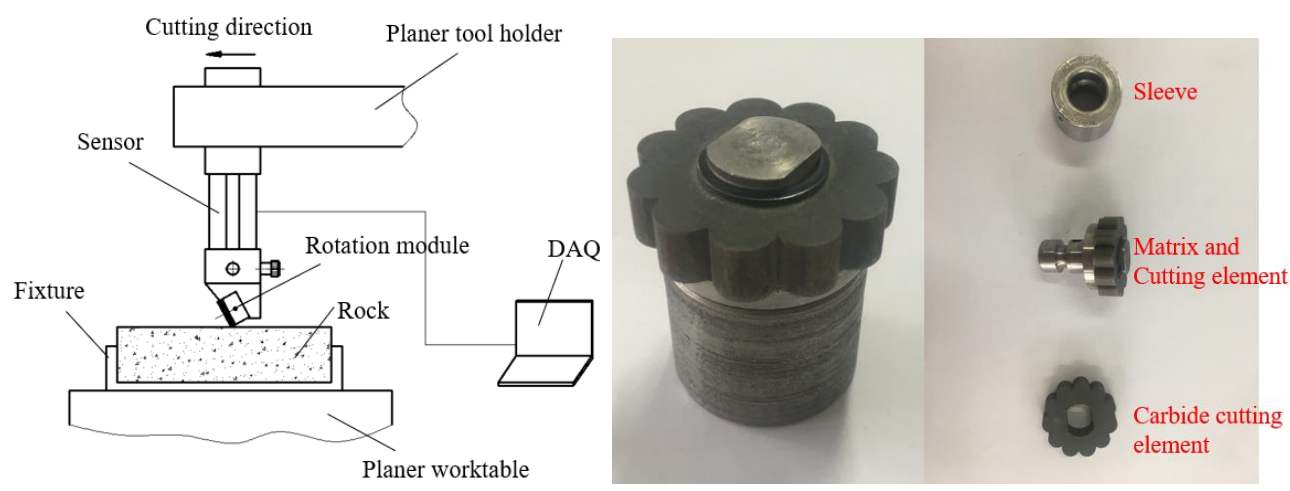

Fig. 6 Scraping experiment of rotating module element Fig. 7 Cutting element of rotating module

Table 1. The primary physical parameters of the rock samples

\begin{tabular}{cccccc}
\hline \multirow{2}{*}{ Rock } & $\begin{array}{c}\text { Uniaxial } \\
\text { compressive } \\
\text { strength }(\mathbf{M P a})\end{array}$ & $\begin{array}{c}\text { Shear } \\
\text { strength } \\
(\mathbf{M P a})\end{array}$ & $\begin{array}{c}\text { Internal } \\
\text { friction } \\
\text { angle }\left(^{\circ}\right)\end{array}$ & $\begin{array}{c}\text { Elastic } \\
\text { modulus } \\
(\mathbf{G P a})\end{array}$ & $\begin{array}{c}\text { Poisson's } \\
\text { ratio }\end{array}$ \\
\hline Sandstone & 67.548 & 13.56 & 38.03 & 11.54 & 0.062 \\
\hline
\end{tabular}

\subsection{Experiment content}

The experiment contents include: different journal angle $\left(0^{\circ}, 5^{\circ}, 10^{\circ}, 15^{\circ}, 20^{\circ}, 25^{\circ}\right.$, $\left.30^{\circ}\right)$, different side angle $\left(0^{\circ}, 5^{\circ}, 10^{\circ}, 15^{\circ}, 20^{\circ}, 25^{\circ}\right)$, different number of teeth $(9,10,11$, 12, 13). The specific experimental content is shown in Table 2 :

Table 2 Experimental Content

\begin{tabular}{llccc}
\hline $\begin{array}{l}\text { Experiment } \\
\text { No. }\end{array}$ & Experimental Content & The penetration & $\begin{array}{c}\text { Cutting speed } \\
\text { depth }(\mathbf{m m})\end{array}$ & $\begin{array}{c}\text { Experiment } \\
\text { Times }\end{array}$ \\
\hline ( $\mathbf{m} / \mathbf{s})$ & 2 & 0.3 & 3 \\
& $\begin{array}{l}\text { The journal angle of the Rotating } \\
\text { module }\left(0^{\circ} \sim 30^{\circ}\right)\end{array}$ & &
\end{tabular}




\subsection{Experimental method and process}

First, the rock sample is placed on the rock clamping tool, then the scraping surface of the rock sample is parallel to the planer table by adjusting the position of the rock sample, the rotating module and the three-way force sensor are fixed on the planer frame, the position of the planer frame is adjusted to make the cutting tooth's eating depth specified in the experiment, the channel of the signal acquisition system is cleared and the sampling is started, and then The testing machine scrapes the rock sample in a straight line, breaks the rock sample, records the experimental data through the data acquisition system, collects the broken rock debris, and completes one experiment. Each experiment is repeated at least three times, and the specific experimental process is shown in Fig. 8.
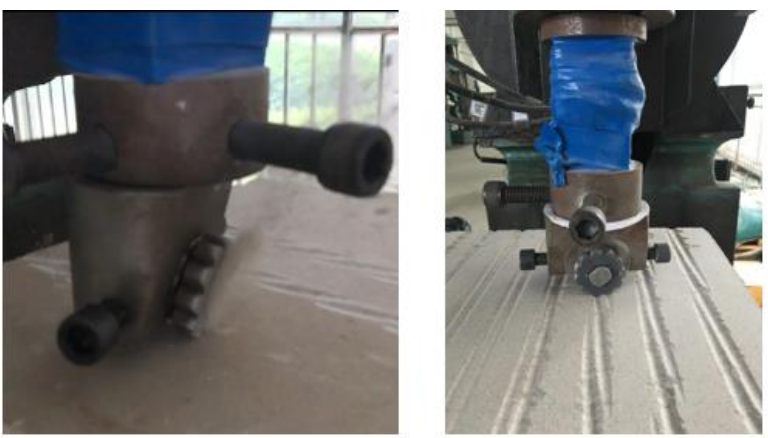

Fig. 8 Experiment process
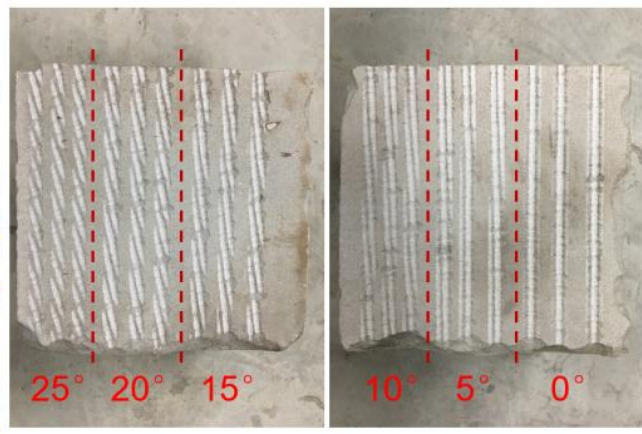

Fig. 9 Craping trace of rotating module element

\subsection{Analysis of experimental results}

The scraping trace of the rotating module element on the rock is different from that of the fixed teeth, and the scraping trace of the different side corners is shown in Fig. 9. It can be seen from the figure that: with the increase of the side corners of the rotating module teeth, the rotating speed of the rotating module teeth increases gradually, and the number of scratches increases gradually. Because the side angle and the angle of the axis of the rotating module element will affect its rotating speed, the relationship between the rotating speed and the journal angle and the side angle is made as shown in Fig. 10. The diameter of the outer circle of the rotating module element $D_{2}=35 \mathrm{~mm}$, the diameter of the small teeth of the module element $D=8 \mathrm{~mm}$, and the number of the small teeth of the module element $n=11$. The scraping experiment is completed on the sandstone. 


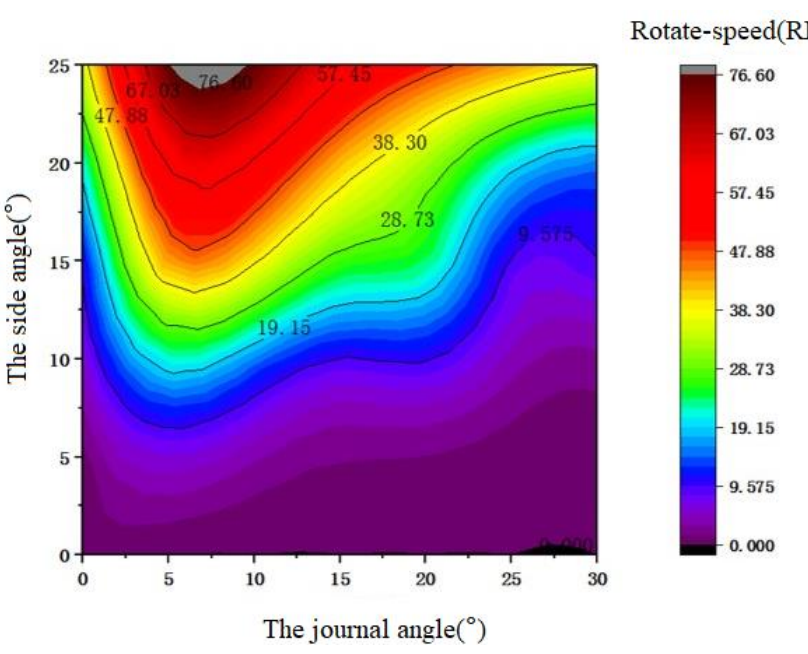

Fig.10 Contour map of rotation module element Rotate-speed

When the side angle is $90^{\circ}$, the rotating module element does pure rolling. When the side angle is $0^{\circ}$, the rotating module element does not rotate and does pure sliding. With the gradual increase of the side angle, the greater the rotating force of the driving module element is. When the side angle increases from $0^{\circ}$ to $90^{\circ}$ under any axle inclination, the rotating speed increases gradually. When the journal angle increases from $0^{\circ}$ to $5^{\circ}$, the rotating speed of the rotating module element tends to increase. The reason is that when the journal angle of the module element is $0^{\circ}$, the tooth cylinder of the module element contacts with the rock, so the module element has to overcome some friction caused by the contact between the tooth cylinder of the module element and the rock when it rotates. When a small journal angle is given to the module element, the tooth surface of the cutting tooth first cuts off the rock, because of a certain journal angle, the cylinder surface of the cutting tooth can avoid too much contact with the rock, reducing the friction; when the journal angle increases from $5^{\circ}$ to $30^{\circ}$, the rotation speed of the single element of the rotating module decreases gradually, because the module element receives the reaction force of the rock along the axis of the module element .The component force of the direction increases gradually, so the force of driving module element to rotate decreases gradually. At the same time, it needs to overcome the friction produced by more module element bearings. Therefore, with the increase the journal angle, the speed decreases gradually.

Because of the principle that the module elements work alternately, the load of the module element is different from that of the fixed PDC teeth. At the same time, the load variation law of the whole module element also has a great influence on the bearing life. The experiment shows that the axial force and tangential force of the rotating module element follow the different side angle conditions The change rule of the journal angle is shown in Fig. 11. It can be seen from the figure that when the journal angle is $10^{\circ}$ and $15^{\circ}$, the axial and tangential loads on the rotating module element are relatively small. At this time, the invasion ability of the rotating module element is better, which is also conducive to extending the service life of the module element. With the increase of the journal angle, the axial and tangential loads on the rotating module element increase, and the invasion ability of the module element decreases. 

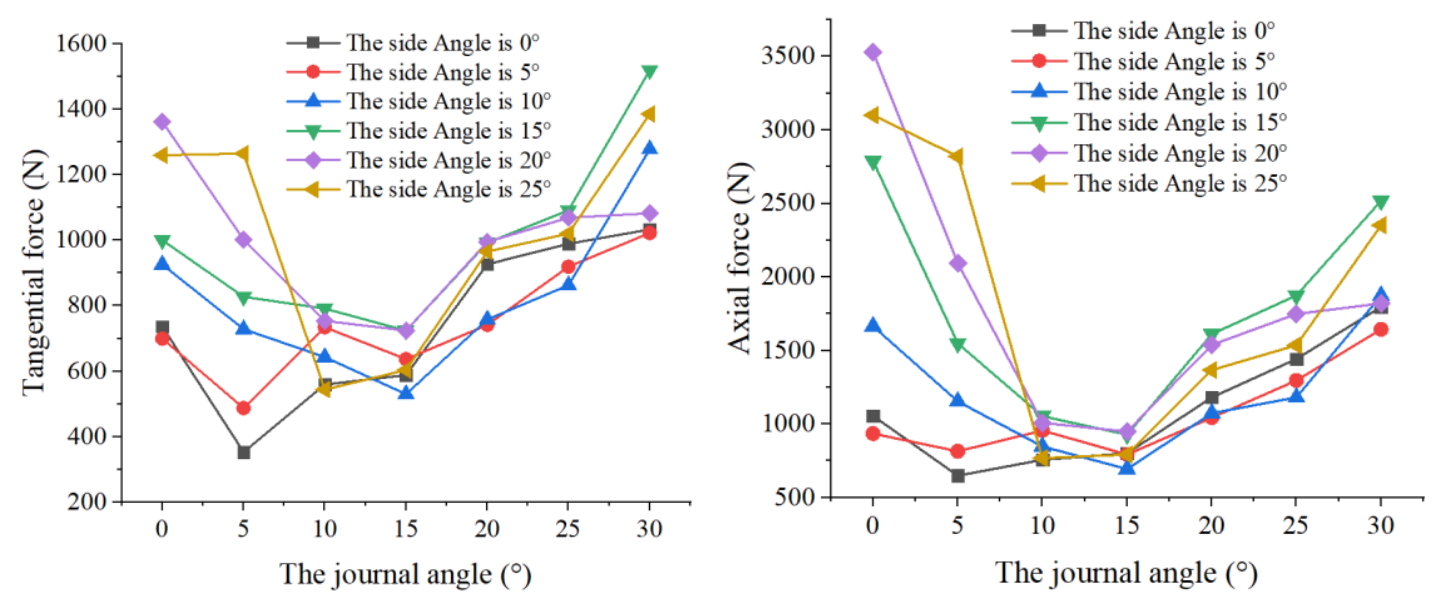

Fig.11 Force of rotating module element

The mechanical specific energy(MSE) of rock, also known as the crushing work per element volume, refers to the energy required to crush the rock per element volume, which is an important index to study the rock breaking efficiency. For the rotating module element, the change rule of its crushing specific work with the side angle and the journal angle is shown in Figure 12 (the diameter of the outer circle of the rotating module element $D_{2}=35 \mathrm{~mm}$, the diameter of the module element pinion $d=8 \mathrm{~mm}$, and the number of the module element pinion $n=11$ ). It can be seen from the figure that: with the increase of the side angle, the crushing specific work of the rotating module element increases gradually, and the rock breaking efficiency decreases gradually; with the increase of the journal angle, the MSE of the rotating module element decreases first and then increases, with the journal angle of $10^{\circ}$, the MSE is smaller, and the rock breaking efficiency is higher.

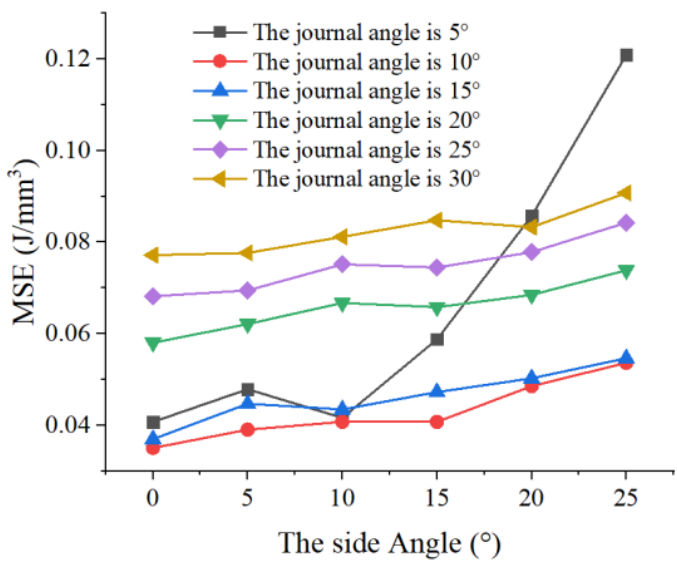

Fig.12 MSE of rotating module element

In order to facilitate observation, the larger the rotation speed of the side angle is higher, and the scraping traces formed on the rock are more obvious. Therefore, the scraping experiments with different tooth distribution density are carried out when the journal angle is $20^{\circ}$ and the side angle is $20^{\circ}$. The cutting depth is $2 \mathrm{~mm}$, the outer circle diameter of the module element is $35 \mathrm{~mm}$, and the diameter of the module single small tooth is $\mathrm{d}=8 \mathrm{~mm}$. The relationship between module element speed and module tooth distribution density is shown in Figure 13. It can be seen from the figure that with the increase of the number of cutting teeth in the module element, the axial force and tangential force of the module element fluctuate. When the number of cutting teeth $n$ is equal to 11, the tangential force and axial force received by the module are the minimum. At this 
time, the rock breaking specific work of the module element is the minimum and the rock breaking efficiency is the highest. In the process of rock cutting, at least two or more cutting teeth of the module element must be able to touch the bottom at the same time, so as to ensure the normal rotation of the module element. When the number of cutting teeth of the module element is small, the spacing between adjacent teeth is too large, resulting in the impact load of the module during the rotation process; with the increase of the number of cutting teeth, resulting in the rapid increase of the rotational speed of the module element In this way, the impact load of the module element increases, which is not conducive to extending the service life of the rotating module element. Therefore, the optimal number of cutting teeth of the rotating module element is to enable the module element to rotate stably and slowly. Therefore, it is necessary to optimize the design when determining the number of cutting teeth of the module element.
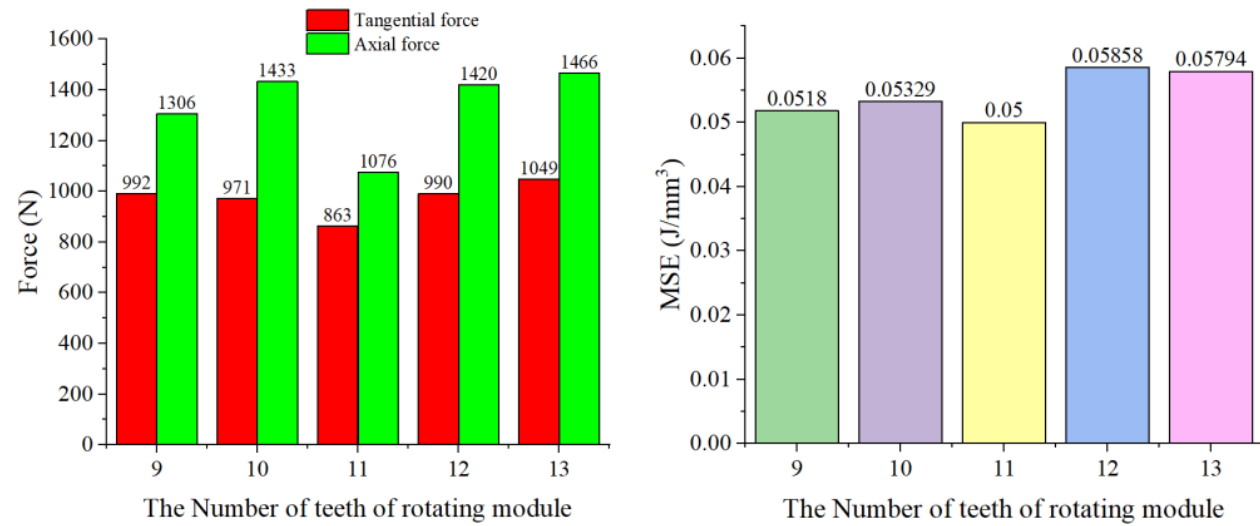

Fig. 13 Operation of rotation module with different number of cutting teeth

\section{Laboratory bench test of rotary modular PDC bit}

\subsection{Design and manufacture of rotary modular PDC bit}

An 8-1/2-inch experimental bit is designed. The number of blades is 4 , the number of rotating module elements is 5 , which are arranged in different radial positions. The diameter of the working contour of the rotating module element is $D_{2}=35 \mathrm{~mm}$, the diameter of the module element teeth is $d=8 \mathrm{~mm}$, and the number of $n$ is 11 . The structural parameters of the rotating module element are selected in combination with the element experiments. the journal angle is $15^{\circ}$, the side angle is $15^{\circ}$, the diameter of the PDC cutting teeth is $13.44 \mathrm{~mm}$, and the height is $8 \mathrm{~mm}$. The experimental drill is shown in Fig.14. The experimental drill controls the rotary cutting of the rotating module cutting element through the pin.
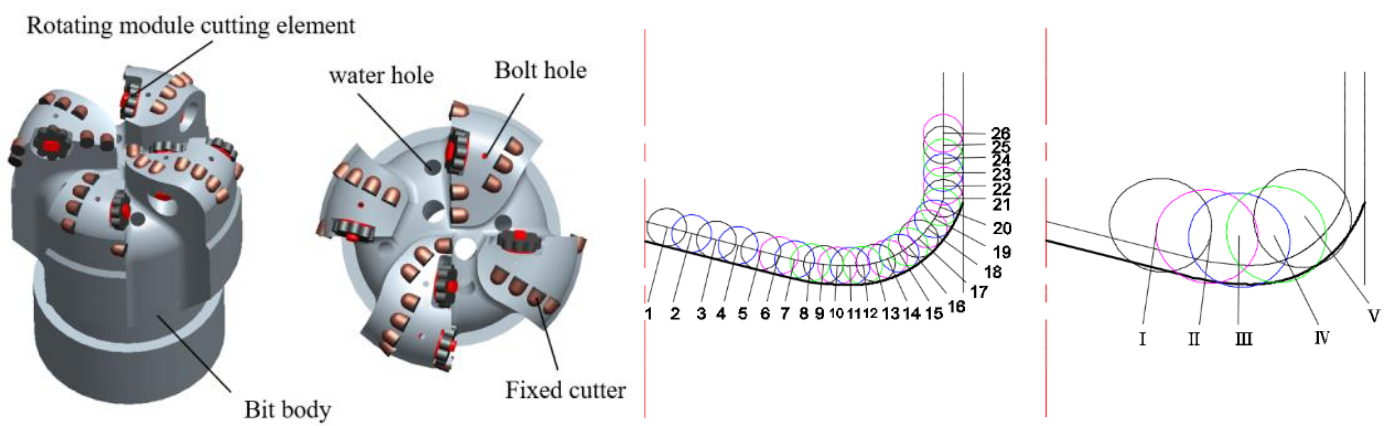

Fig. 14 three dimensional diagram and tooth arrangement diagram of experimental bit

The machining of the cutting teeth of the bearing and the rotating module element adopts the common lathe and wire cutting to weld the cutting teeth on the fixed wing and the body joint. 

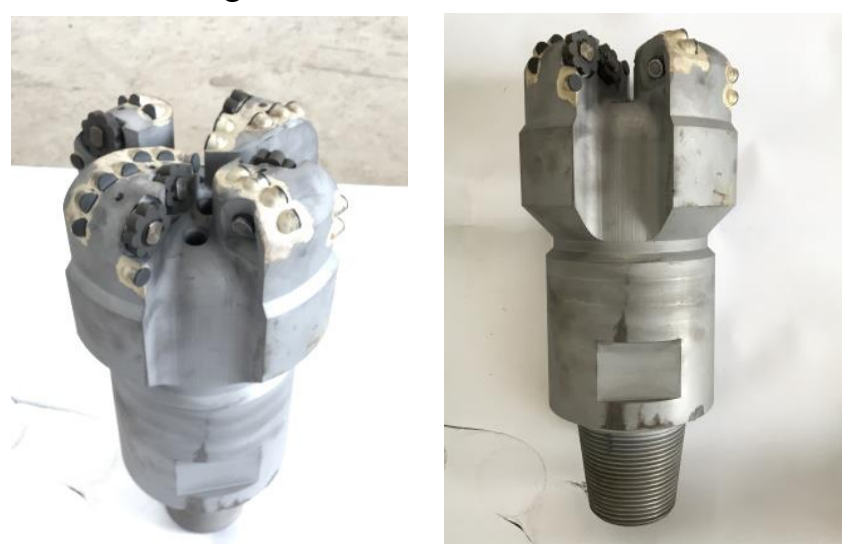

Fig. 15 Photo of experimental bit

\subsection{Laboratory bench test process and method}

In the experiment, By the comparison and analysis of bottom hole model, the bit pressure fluctuation, torque and mechanical drilling speed during drilling, the rock breaking law PDC was studied. The bit slowly drills down, completes the bottoming work of rock samples, starts the lower turntable and applies bit pressure to make the bit drill normally (as shown in Fig. 16), starts the dynamic test analysis system, and performs channel balance and zeroing, collects experimental data, after reaching predetermined drilling depth, stops drilling and collects cutting. Each set of experiments is repeated at least 3 times.
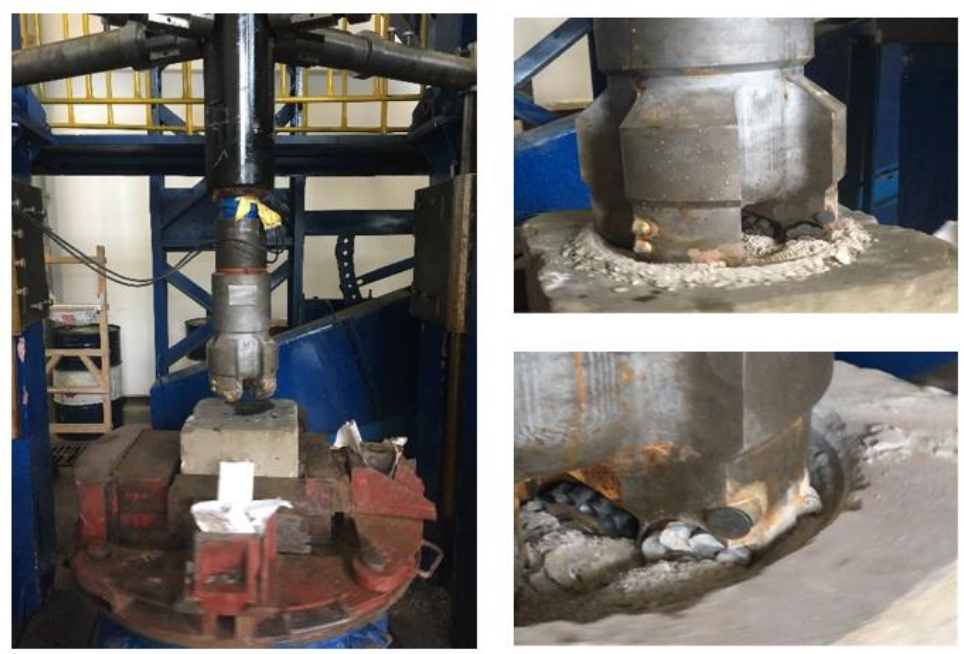

Fig.16 Experiment process

\subsection{Analysis of experimental results}

During the experiment, it is clearly observed that the module element rotates slowly at the bottom of the well. The bottom of the well morphology obtained from the experiment is shown in Fig.17. Because the relative height between the module element and the fixed cutting teeth in the experimental bit is small, there is no local cross scraping track at the bottom of the well; however, since the module element 5 is set at the hole diameter maintaining position of the bit, it can scrape the well wall, in the red circle area in the figure There are traces formed after scraping the rotary module, which also proves from the side that the rotary module element can rotate stably at the bottom of the well, scraping the rock. 

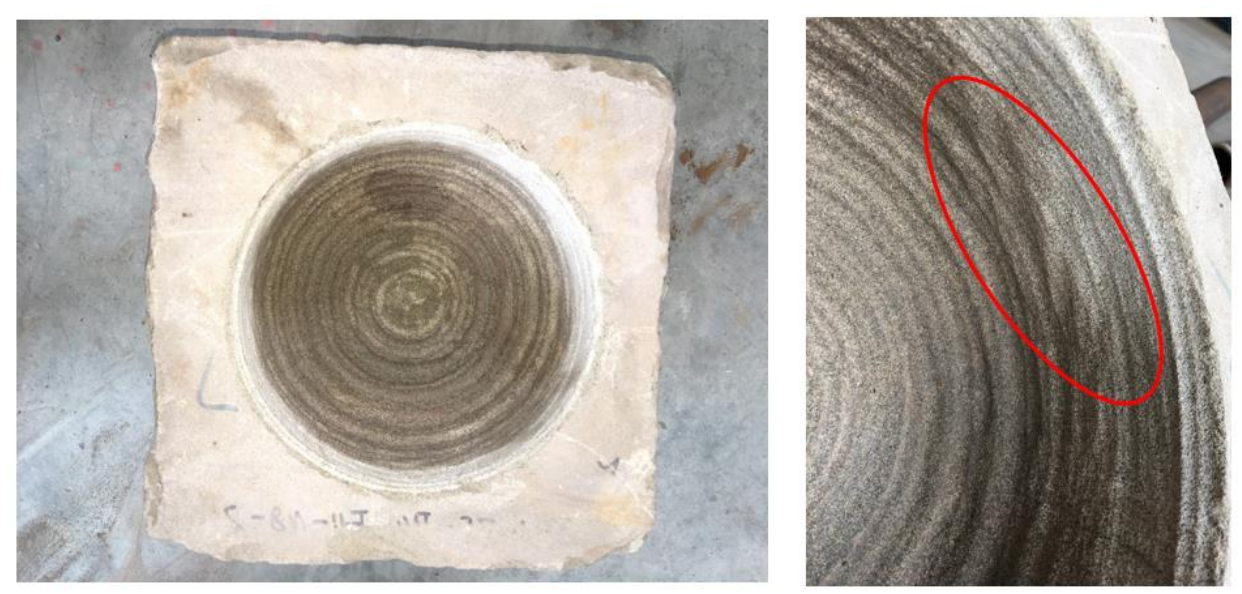

Fig.17 Bottom hole morphology after the experiment

The experimental bit controls the rotation of the rotating module element through the pin. When the module element does not rotate, the experimental bit is a conventional PDC bit. When the module rotates, the experimental bit is a new type of rotating module PDC bit. Fig. 18 is a comparison ROP and MSE of two kinds of bits in sandstone when the WOB is $10 \mathrm{kN}$ and the rotating speed is 10rpm. It can be seen from the chart that: compared with the conventional PDC bit, the mechanical ROP of rotary modular PDC bit is $1.09 \mathrm{~m} / \mathrm{h}$, which increases by $15 \%$; the MSE is $0.41 \mathrm{j} / \mathrm{m} 3$, which decreases by $14 \%$. It shows that the rotary modular element can achieve local cross scraping and reduce rock breaking energy consumption, Improve the rock breaking efficiency of the bit.
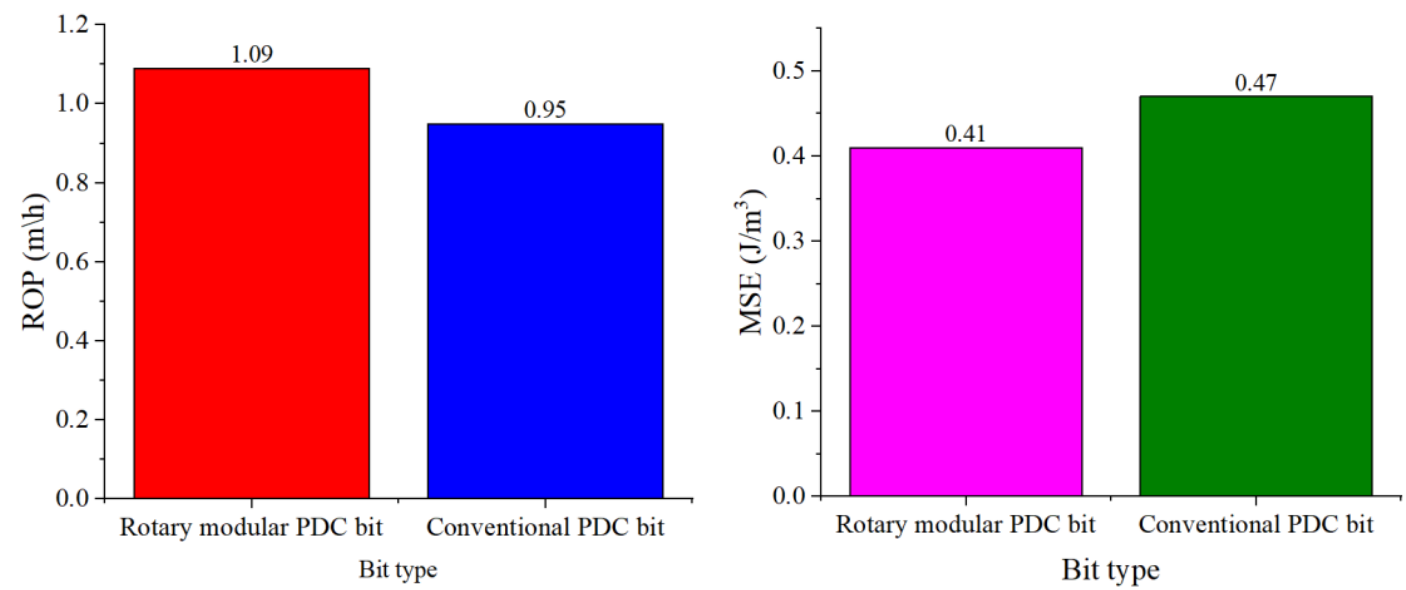

Fig. 18 Comparison of ROP and MSE of two kinds bits

\section{Conclusion}

(1) A new type of PDC bit with rotating module can cool the cutting teeth, reduce the thermal wear of the cutting teeth, and extend the life of the cutting teeth. At the same time, in the radial coverage area of the rotating module element, there is local cross compound scraping, which can improve the invasion ability of the cutting teeth and improve the rock breaking efficiency.

(2) Through the element experiment, it is known that the rotation speed of the rotating module increases with the increase of the side angle, and decreases with the increase of the shaft angle; with the increase of the shaft angle, the cutting load of the rotating module decreases first and then increases, and with the increase of the side angle, the mechanical specific energy of the rotating 
module increases gradually. When t the journal angle is $10^{\circ} \sim 15^{\circ}$, the rock breaking energy consumption of the rotating module element is higher Low, when the side angle is $10^{\circ} \sim 15^{\circ}$, the rotation module element can realize stable low-speed rotation.

(3) Compared with the conventional PDC bit, the new rotary modular PDC bit has a $15 \%$ increase in ROP and a 14\% decrease in MSE.

\section{Acknowledgements}

Funding: This work was supported by the Sichuan Science and Technology Program [Grant No. 2019YJ0537]; China Postdoctoral Science Foundation [Grant No. 2019M653479]; and National Natural Science Foundation of China [Grant No. 51504209].

\section{References}

Zhu H.Y., Deng, J.G., Jin, X.C., Hu, L.B., Luo B., 2015. Hydraulic fracture initiation and propagation of oil and gas wellbore with oriented perforating technique. Rock Mechanics and Rock Engineering, 48, 585-601.

Zhu H.Y., Shen, J.D., Zhang, F.S., 2019. A fracture conductivity model for channel fracturing and its implementation with Discrete Element Method. Journal of Petroleum Science and Engineering, 172, 149-161.

Yingxin Yang, Chunliang Zhang, Lian Chen and Yong Liu.2017.Kinematic and bottom-hole pattern analysis of a composite drill bit of cross-scraping. Proceedings of the Institution of Mechanical Engineers, Part C: Journal of Mechanical Engineering Science.231:3104-3117.

Yingxin Yang, Chunliang Zhang, Yong Liu, Min Lin and Lian Chen. 2018. Research on rock-breaking mechanism of cross-cutting PDC bit. Joural of Petroleum Science and Engineering.161:657-666.

Dan Scott. 2015.A bit of history: Overcoming early setbacks, PDC bits now drill 90\%-plus of worldwide footage[J]. Drilling contractor, IADC, (7/8).

Luc Perneder, Emmanuel Detournay, Geoff Downton,2012.Bit/rock interface laws in directional drilling[J], International Journal of Rock Mechanics and Mining Sciences;51:81-90.

H. Santos, J.C.R. Placide, J.E.Oliveira., 2000. Overcoming Hard rock dilling challenges[J].SPE 59182.

Jiang, M.H., Chen S.X., Zhang, H.Q., 2009. Drilling technology for exploration of solid mineral resources (Volume 1) $[\mathrm{M}]$. Shandong science and technology press.

Mostofi M., Franca L.F.P., Richard T., 2013. Drilling response of impregnated diamond bits: an experimental investigation. U. S. Rock Mechanics/Geomechanics Symposium.

Li, M., 2017. Research on impregnated diamond bit with bionic special-shaped teeth without hard matrix [D]. Jilin University.

Li, M., Su, Y. N., Sun, Y. H., Gao, K., 2016. Diamond bit impregnated with bionic profiled teeth and high matrix [J]. Journal of Jilin University (Engineering Edition), 46(05):1540-1545.

Platt J, Valliyappan S, Karuppiah V, 2016. Innovative Rolling Cutter Technology Significantly Improved Footage and ROP in Lateral and Vertical Gas Applications in Saudi Arabia[C]//SPE/IADC Middle East Drilling Techn ology Conference and Exhibition.Society of Petroleum Engineers .1-8. 